\title{
Distribution of Hepatitis Delta Virus Genotypes in Mashhad, Northeast Iran
}

\author{
Hamid Sadeghian 1; Naiemeh Varasteh ²; Abbas Esmaeelzadeh 3; Hosein Nomani 4; Maliheh \\ Alimardani 1; Mahdieh Davoodnejad 5; Mojtaba Meshkat 6; Mitra Ahadi 3; Samaneh Sepahi 7; \\ Sina Rostami 8; Zahra Meshkat ${ }^{5, *}$ \\ ${ }^{1}$ Department of Laboratory Sciences, School of Paramedical Sciences, Mashhad University of Medical Sciences, Mashhad, IR Iran \\ ${ }_{2}^{2}$ School of Medicine, Islamic Azad University, Mashhad Branch, Mashhad, IR Iran \\ ${ }_{3}^{3}$ Department of Internal Medicine, School of Medicine, Mashhad University of Medical Sciences, Mashhad, IR Iran \\ ${ }_{5}^{4}$ Antimicrobial Resistance Research Center, Mashhad University of Medical Sciences, Mashhad, IR Iran \\ 5 Antimicrobial Resistance Research Center, Mashhad University of Medical Sciences, Mashhad, IR Iran \\ 6 Department of Human Genetic, School of Medicine, Mashhad University of Medical Sciences, Mashhad, IR Iran
6 Department of Biostatistics, School of Allied Medical Sciences, Shahid Beheshti University of Medical Sciences, Tehran, IR Iran \\ 7 Targeted Drug Delivery Research Center, School of Pharmacy, Mashhad University of Medical Sciences, Mashhad, IR Iran \\ 8 Department of Biology, Faculty of Sciences, Ferdowsi University of Mashhad, Mashhad, IR Iran \\ ${ }^{*}$ Corresponding author: Zahra Meshkat, Antimicrobial Resistance Research Center, Mashhad University of Medical Sciences, Mashhad, IR Iran. Tel: +98-5118012453, Fax: +98- \\ 5118002960, E-mail: meshkatz@mums.ac.ir
}

Received: September 17, 2013; Revised: November 17, 2013; Accepted: December 25, 2013

\begin{abstract}
Background: Hepatitis delta virus (HDV) is dependent on the hepatitis B virus for transmission and propagation. Based on isolated HDV sequences from different parts of the world, at least three major different genotypes with different geographic distributions are suggested. Studies have shown that genotype 1 is the predominant genotype of HDV in different parts of Iran; however, the genotype distribution of this virus has not been identified in Mashhad, northeast Iran.

Objectives: This current study determines the frequency of HDV major genotypes in Mashhad, Iran.

Patients and Methods: Twenty-five participants were enrolled in this study. All samples were positive for HBsAg (determined by Enzymelinked immunosorbent assay (ELISA)) and anti-HDV. RNA extraction and cDNA synthesis was performed. Then, PCR was performed and HDV genotypes were determined by restriction fragment length polymorphism (RFLP).

Results: Of 25 patients, 12 (48\%) were positive for HDV RNA. Genotype analysis of HDV RNA revealed that the prevalence of HDV genotypes I and II was $83.3 \%(n=10)$ and $16.7 \%(n=2)$, respectively.

Conclusions: This study showed that the most prevalent genotype of HDV in Mashhad was genotype I. It was of interest that in contrast to other provinces of Iran, HDV genotype 2 was observed in Mashhad. Similar studies with larger sample sizes could provide valuable information regarding the molecular epidemiology and geographical distribution. It may also help control and prevent the spread of hepatitis D virus infections. In addition, the genotyping of HDV may predict the severity of the disease.
\end{abstract}

Keywords:Hepatitis Delta Virus; Genotype; Polymerase Chain Reaction; Restriction Fragment Length Polymorphism

\section{Background}

Hepatitis delta virus (HDV) was first identified by Rizzetto (1). The presence of Pro-205 in HDV genotype 1 may account for its higher assembly efficiencies and wider distribution (2). New studies suggest at least eight HDV clades, which are phylogenetically distinct and have predominance in different regions worldwide. Clade 1 of HDV is found in all regions. However, Clade 2 is mostly reported from Japan, Taiwan, and Russia. Clade 4 is localized to Taiwan and Japan. Clade 3 has the highest prevalence in the Amazonian region while HDV clades 5, 6, 7 and 8 are found in Africa (3). The epidemiology of HDV infection has been classified into three categories as follows: 1. the endemic pattern that exists in countries such as Greece and Italy; 2. the epidemic pattern that is observed in Venezuela; and 3. the occurrence of HDV infection that is found among intravenous drug users in developed countries (4).
The endemic pattern of HDV infection exists in the Middle East (4). Globally, HDV infection is found in more than 15 million people. Its prevalence is mostly focused in Italy, Eastern Europe, and western Asia (5). Recently, a study reported that there are about 350 million carriers of $\mathrm{HBV}$ infection worldwide of which 18 million are infected with HDV (6). Countries such as Iran and Pakistan showed an increase in HDV prevalence. On the other hand, countries like Turkey, India, Australia, China, Japan, and Taiwan, which had a very high HDV prevalence in the past, have shown a decline in the incidence rate, although a high prevalence rate persists in some of them (6). Alavian et al. reported the prevalence of HDV infection in different provinces of Iran. They reported the prevalence rate of 9.7\% in Fars, 2\% in Yazd; 11.5\% in HBsAg positive subjects and $63.7 \%$ among cirrhotic patient in Khuzestan; $20.7 \%$ in Kerman; 13.8\% in Sistan and Balochestan; 5.8\% in Goles-

Copyright (C) 2015, Ahvaz Jundishapur University of Medical Sciences. This is an open-access article distributed under the terms of the Creative Commons Attribution-NonCommercial 4.0 International License (http://creativecommons.org/licenses/by-nc/4.0/) which permits copy and redistribute the material just in noncommercial usages, provided the original work is properly cited. 
Meshkat M et al.

tan; 2.4-5\% in Tehran; 9.3\% in East Azarbaijan; and 31.37\% in patients positive for HBV and HDV infections in Kerman$\operatorname{shah}(8,9)$.

In Iran, the prevalence of HDV is considered to have declined from $18.03 \%$ in 1990 to $5.7 \%$ in 2005. However, HbsAg positivity is a serious risk for HDV infection $(7,9,10)$. Malekzade et al. reported the first case of HDV infection in Iran (11). It occurred as a co-infection or superinfection with hepatitis B virus (HBV). The persistent replication of HBV and HDV was associated with more severe outcomes. Over $90 \%$ of patients who are superinfected are likely to develop severe conditions such as cirrhosis or hepatocellular carcinoma. Coinfection with $\mathrm{HBV}$ is responsible for more cases of acute liver disease and fulminant hepatitis (70-80\% of the total cases) (12-14), while it evolved to chronicity in only $2 \%$ of cases (14).

Longitudinal follow-up studies have indicated that the HDV genotype 1 is the most critical factor associated with survival rate along with severity and progression of liver failure $(1,15)$. Genotype 2 is associated with a milder hepatitis and 2a is more pathogenic (16). Genotype 3 often leads to fulminant hepatitis (16). The frequency of HDV genotypes and clades were undetermined in the city of Mashhad. In consideration of the geographical vastness of Mashhad, which is home to many immigrants from Afghanistan as well as being a city of pilgrimage attracts many people from different regions of Iran and other middle-eastern countries. There, it is expected that the pattern of prevalence is different from other parts of the country.

\section{Objectives}

This study was performed to determine the frequency of HDV genotypes in the city of Mashhad, Iran.

\section{Patients and Methods}

Twenty-five subjects were enrolled in this study. Informed consent was obtained and a questionnaire was filled for each participant. All samples were positive for HBsAg and anti-HDV antibody. This was confirmed by ELISA (HDV Ab ELISA kit, Delaware Biotech, USA).

\subsection{RNA Extraction and cDNA Synthesis}

One hundred and forty microliter of sera was collected for RNA extraction using the QIAamp viral RNA mini kit (Qiagen, USA) according to the manufacturer's recommended procedures. Extracted RNA was eluted in $60 \mu \mathrm{L}$ of elusion buffer. cDNA synthesis was performed with an Omniscript RT kit (Qiagen, USA) according to the manufacturer's instructions. HDV genotyping HDV genotyping was performed using a semi-nested PCR method. D120 forward primer (5'-ATGCCATGCCGACCCGAAGAGGAA-3'), DH1 (5'-GGCCTCTCAGGGGAGGATTCAC-3'), and D118 (5'-CTCAGGGGAGGATTCACCGACA-3') reverse primers were used in the semi-nested PCR, as described by Mirshafiee et al. (12). Briefly, for the first round of PCR amplification, the mixture contained $10 \mathrm{pmoL}$ of each D120 and DH1 primers, and $10 \mathrm{mM}$ dNTP and 5U Taq DNA polymerase (Cinna Gen, Iran). For the second round of PCR, $2 \mu \mathrm{L}$ of the first-round PCR products was added to $10 \mathrm{pm}$ of each D120 and D118 primers, and $10 \mathrm{mM}$ dNTP and 5U Taq DNA Polymerase. PCR products were subjected for restriction enzyme digestion using SmaI and XhoI restriction endonucleases.

\section{Results}

Of the 25 subjects, 9 were female and 16 were male. The mean age was $47 \pm 10.14$. In total, 12 cases $(48 \%)$ were positive for HDV RNA. Of these 12 patients, 4 were female and 8 were male. All 12 patients were living in Mashhad when the study was performed; however, three patients were born in Afghanistan. At least 4 of the 12 patients had a family history of HDV infection. For HDV genotype 1, digestion with SmaI produced fragments of 219 and $222 \mathrm{bp}$. While with XhoI, fragments of 382 and 59 bp were produced (Figure 1). SmaI does not have any restriction site for the amplified fragments of HDV genotype 2. XhoI produces three fragments of $81 \mathrm{bp}, 301 \mathrm{bp}$, and $59 \mathrm{bp}$ (Figure 1). Genotype analysis of HDV RNA showed that the prevalence of HDV genotype 1 and 2 was $83.3 \%(n=10)$ and $16.7 \%$ $(\mathrm{n}=2)$, respectively.

Figure 1. Results of PCR-RFLP for Four Samples of Patients with HDV Infection

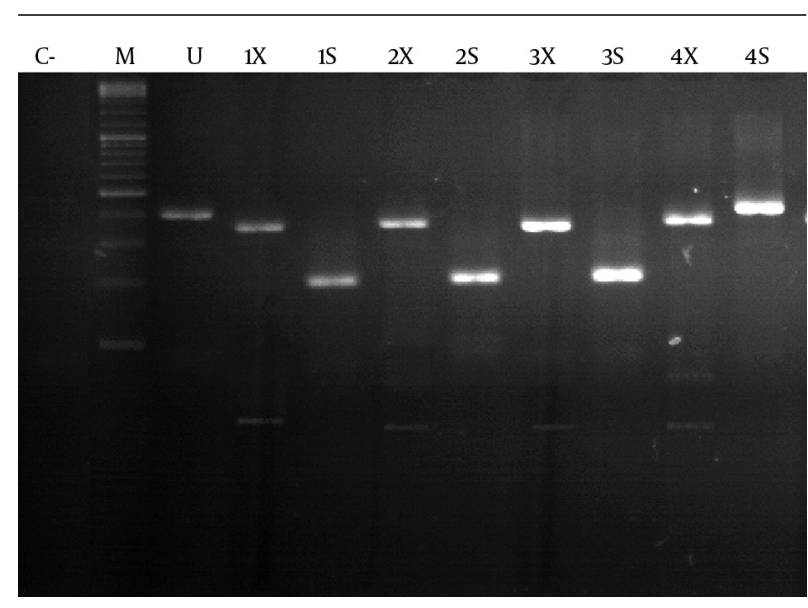

1, 2, and 3 represent samples infected with HDV genotype one and 4 represents sample infected with HDV genotype 2. M:100 bp DNA Size Marker; U: Undigested PCR Product; X: XhoI digestion; S: SmaI Digestion. C- is control negative.

\section{Discussion}

The genotyping of HDV could be performed using restriction fragment length polymorphism of PCR products (17), sequencing, and staining of liver biopsies with genotype-specific antibodies (18). In the Middle East, the predominant genotypes of HBV and HDV are D and 
Meshkat M et al.

1, respectively. The association of HDV genotype 1 with HBV genotype D was reported in Pakistan, Turkey, Egypt, and Italy (19-21). Genotype 1 is reported to be the most prevalent genotype in different parts of Iran $(12,22,23)$. Mohebbi et al. indicated that 25 patients from different parts of Iran who were positive for HDV antibody were as follows: 22 (88\%) were HDV RNA positive and their phylogenetic analysis showed that all of them were infected by genotype 1 (clade 1). (23). Mirshafiee et al. indicated that 35 patients with HBsAg and anti-HDV positivity in Tehran. Among them, 13 (38.46\%) cases were positive for HDVRNA. They performed RT-semi-nestd PCR and RFLP and found that all cases were of genotype 1 (12).

Khalafkhany et al. showed that 31 anti-HDV seropositive patients in Iran showed that 15 (48.4\%) were positive for HDV RNA and all belonged to genotype 1 (24). Regarding the neighboring countries of Iran, Perveen et al. performed indicated that in Karachi (Pakistan) that 22 patients all belonged to genotype 1 of HDV (25). In Turkey, HDV1 infection was reported to be endemic among HBsAg carriers, especially in southeastern Turkey. Phylogenetic analysis in 9 Turkish patients who were positive for anti-HDV showed that they were infected with genotype 1(26). In other countries, analyses of HDV genotypes from 29 infected patients living in Ivaniushina showed a frequency of $48.27 \%$ and $51.72 \%$ for HDV genotypes 1 and 2, respectively (27). Also, Neverov et al. showed there was based on the phylogenic analysis of 66 patients in Russia and showed the same frequency (50\%) for genotypes 1 and 2 (28).

A unique HDV genotype 2 in the Miyako Islands was first introduced for 6 patients with HDV-related chronic liver disease. This genotype showed low homology (75-81\%) to the HDV genotype 2 reported from Japan but had a high identity (83-95\%) with the novel genotype 2 (HDV genotype 2 b), which was recently reported from Taiwan (29). A study in the Amazon region of Colombia on HBV and HDV sequences from 7 patients with fulminant hepatitis showed that five were positive for HBV-DNA and HDVRNA. Of these 7 patients, 5 patients were positive for HDV genotype 3 (30). Among $233 \mathrm{HBsAg}$ positive subjects in Cameroon, anti-HDV antibody was found in $17.6 \%$. In addition, phylogenetic analyses showed the presence of HDV clades 1, 5, 6, and 7 (3). Barros et al. evaluated the seroprevalence of HDV among HBsAg chronic carriers in the Northeast Brazil. Among the studied 133 patients, 5 were positive for anti-HDV, of whom 3 were HDV RNA positive. HDV Clades 3 and 8 were found in 1 and 2 patients, respectively (31). Up to now, the epidemiology of HDV has not been comprehensively studied in Iran and limited data is available with regard to its genotypes, clades, and subgroups distribution.

This study was performed for the first time in Mashhad. Genotype analysis of HDV RNA showed that the prevalence of HDV genotypes 1 and 2 was $83.3 \%(n=10)$ and $16.7 \%(n=2)$, respectively. In addition, the findings indicate that the distribution of genotype 2 is not restricted to Taiwan, Japan, and the Miyako Islands and has spread over the Northern Asia in Russia. It is of interest that the two patients infected with HDV genotype 2 in the current study were not from Taiwan or Japan, where this genotype is more prevalent, and had not traveled to any of these countries, although, one of them had traveled to Saudi Arabia. This finding contradicts most studies carried out in Iran and in neighboring countries to Iran including Turkey and Pakistan, which showed no evidence of HDV genotype 2. These two patients were born and lived in Khorasan Razavi province (the province that includes Mashhad).

It is assumed that HDV infection in these regions has a homogenous origin or has become almost similar because of migration. The number of participants was not enough and further studies in this region with larger sample sizes are essential. In addition, the author's encourage further studies in this region to determine the frequency of different HDV clades. Genotyping HDV helps us to determine its molecular epidemiology, geographical distribution, and identification of transmission chains.

\section{Acknowledgements}

The authors would like to thank Ms. Malihe Najib Bagherzadeh for her kind assistance in blood sampling.

\section{Authors' Contributions}

Hamid Sadeghian: Conception and design of the study, analysis, and interpretation of the data. Naiemeh Varasteh: Performing laboratory tests. Abbas Esmaeelzadeh: Clinical evaluation of HDV infected participants. Hossein Nomani: Performing laboratory tests. Maliheh Alimardani: Performing laboratory tests. Mahdieh Davoodnejad: Performing laboratory tests. Mojtaba Meshkat: Conception and design of the study. Mitra Ahadi: Clinical evaluation of HDV infected participants. Samaneh Sepahi and Sina Rostami: Performing laboratory tests. Zahra Meshkat: Conception and design of the study, guarantor of integrity of the entire study.

\section{Funding/Support}

This study was financially supported by the Research Council of Mashhad University of Medical Sciences, Mashhad, Iran.

\section{References}

1. Rizzetto M, Verme G, Recchia S, Bonino F, Farci P, Arico S, et al. Chronic hepatitis in carriers of hepatitis B surface antigen, with intrahepatic expression of the delta antigen. An active and progressive disease unresponsive to immunosuppressive treatment. Ann Intern Med. 1983;98(4):437-41.

2. Shih HH, Shih C, Wang HW, Su CW, Sheen IJ, Wu JC. Pro-205 of large hepatitis delta antigen and Pro-62 of major hepatitis B surface antigen influence the assembly of different genotypes of hepatitis D virus. J Gen Virol. 2010;91(Pt 4):1004-12.

3. Foupouapouognigni Y, Noah DN, Sartre MT, Njouom R. High 
prevalence and predominance of hepatitis delta virus genotype 1 infection in Cameroon. J Clin Microbiol. 2011;49(3):1162-4.

4. Alavian SM, Alavian SH. Hepatitis D Virus Infection; Iran Middle East and Central Asia. Hepat Mon. 2005;5(4):137.

5. Mumtaz K, Hamid SS, Adil S, Afaq A, Islam M, Abid S, et al. Epidemiology and clinical pattern of hepatitis delta virus infection in Pakistan.J Gastroenterol Hepatol. 2005;20(10):1503-7.

6. Abbas Z, Jafri W, Raza S. Hepatitis D: Scenario in the Asia-Pacific region. World J Gastroenterol. 2010;16(5):554-62.

7. Amini S, Mahmoodi MF, Andalibi S, Solati AA. Seroepidemiology of hepatitis B, delta and human immunodeficiency virus infections in Hamadan province, Iran: a population based study.JTrop Med Hyg. 1993;96(5):277-87.

8. Alavian. S., Asari. S., Manzori-Joybari H. Prevalence and risk factors of HDV infection in HBV infected cases. Govaresh. 2004;9:217-21.

9. Alavian SM, Assari S, Manzoori-Joybari H, Moghani Lankarani M, Doroudi T, Haji-Beigi B, et al. Frequency and risk factors of hepatitis D virus in hepatitis B patients. Govaresh. 2012;10(1):21-6.

10. Rezvan H, Forouzandeh B, Taroyan S, Fadaiee S, Azordegan F. A study on delta virus infection and its clinical impact in Iran. Infection. 1990;18(1):26-8.

11. Malekzadeh R, Borhanmanesh F. Prevalence of HDV in asymptomatic healthy carrier of HBV in Iran. Irn J Med Sci. 1989;14(2):33-8.

12. Mirshafiee H, Mahmoodian-Shooshtari M, Sharifi Z, Hosseini SM. Genotype analysis of hepatitis delta virus from hepatitis B surface antigen-positive patients using PCR-RFLP in Tehran, Iran. Arch Iran Med. 2009;12(3):238-43.

13. Wu JC. Functional and clinical significance of hepatitis D virus genotype II infection. Curr Top Microbiol Immunol. 2006;307:17386.

14. Farci P, Niro GA. Clinical features of hepatitis D. Semin Liver Dis. 2012;32(3):228-36.

15. Smedile A, Farci P, Verme G, Caredda F, Cargnel A, Caporaso N, et al. Influence of delta infection on severity of hepatitis B. Lancet. 1982;2(8305):945-7.

16. Nakano T, Shapiro CN, Hadler SC, Casey JL, Mizokami M, Orito E, et al. Characterization of hepatitis D virus genotype III among Yucpa Indians in Venezuela.J Gen Virol. 2001;82(Pt 9):2183-9.

17. Wu JC, Choo KB, Chen CM, Chen TZ, Huo TI, Lee SD. Genotyping of hepatitis D virus by restriction-fragment length polymorphism and relation to outcome of hepatitis D. Lancet. 1995;346(8980):939-41.

18. Hsu SC, Syu WJ, Ting LT, Wu JC. Immunohistochemical differentiation of hepatitis D virus genotypes. Hepatology. 2000;32(5):1111-6.

19. Bozdayi AM, Aslan N, Bozdayi G, Turkyilmaz AR, Sengezer T, Wend
$\mathrm{U}$, et al. Molecular epidemiology of hepatitis B, C and D viruses in Turkish patients. Arch Virol. 2004;149(11):2115-29.

20. Saudy N, Sugauchi F, Tanaka Y, Suzuki S, Aal AA, Zaid MA, et al. Genotypes and phylogenetic characterization of hepatitis B and delta viruses in Egypt. J Med Virol. 2003;70(4):529-36.

21. Niro GA, Smedile A, Andriulli A, Rizzetto M, Gerin JL, Casey JL. The predominance of hepatitis delta virus genotype I among chronically infected Italian patients. Hepatology. 1997;25(3):728-34.

22. Behzadian F, Sabahi F, Karimi M, Sadeghizadeh M, Maghsoudi N Forooshani RS, et al. Molecular phylogenetic analysis of Iranian HDV complete genome. Virus Genes. 2005;30(3):383-93.

23. Mohebbi SR, Zali N, Derakhshan F, Tahami A, Mashayekhi R, Amini-Bavil-Olyaee S, et al. Molecular epidemiology of hepatitis delta virus (HDV) in Iran: a preliminary report. J Med Virol. 2008;80(12):2092-9.

24. Khalafkhany D, Makvandi M, Javanmard D, Hamidi-Fard M, Samarbaf-Zadeh A. Determination of Hepatitis Delta Virus Genotype among HBV Carriers in Southwest of Iran. Iran J Virol. 2013;6(1):6-12.

25. Perveen S, Nasir MI, Shahid SM, Azhar A, Khan OY. Phylogenetic analysis of HDV isolates from HBsAg positive patients in Karachi, Pakistan. Virol J. 2012;9:162.

26. Celik I, Karataylı E, Cevik E, Kabakci SG, Karatayli SC, Dinc B, et al. Complete genome sequences and phylogenetic analysis of hepatitis delta viruses isolated from nine Turkish patients. Arch Virol. 2011;156(12):2215-20.

27. Ivaniushina V, Radjef N, Alexeeva M, Gault E, Semenov S, Salhi M et al. Hepatitis delta virus genotypes I and II cocirculate in an endemic area of Yakutia, Russia.J Gen Virol. 2001;82(Pt 11):2709-18.

28. In: Neverov AD, Karandashova IV, Rafailova MA, Braslavskaya SI, Alekseyeva MN, Chulanov VP editors. Genetic Heterogeneity of Hepatitis B and D Viruses in Yakutia (Russia). Russia. 2006 13th International Congress on Circumpolar Health Gateway to the International Polar Year. pp. 12-6.

29. Sakugawa H, Nakasone H, Nakayoshi T, Kawakami Y, Miyazato S, Kinjo F, et al. Hepatitis delta virus genotype IIb predominates in an endemic area, Okinawa, Japan. J Med Virol. 1999;58(4):366-72.

30. Alvarado-Mora MV, Romano CM, Gomes-Gouvea MS, Gutierrez MF, Carrilho FJ, Pinho JR. Dynamics of hepatitis D (delta) virus genotype 3 in the Amazon region of South America. Infect Genet Evol. 2011;11(6):1462-8.

31. Barros LM, Gomes-Gouvea MS, Pinho JR, Alvarado-Mora MV, Dos Santos A, Mendes-Correa MC, et al. Hepatitis Delta virus genotype 8 infection in Northeast Brazil: inheritance from African slaves? Virus Res. 2011;160(1-2):333-9. 